\title{
Molecular study of Pompe disease in Egyptian infants
}

\author{
Mona Essawi ${ }^{1}$, Nagham ElBagoury' ${ }^{1}$ Engy Ashaat ${ }^{2}$, Wessam Sharaf-Eldin ${ }^{1 *}$ [C and Ekram Fateen ${ }^{3}$
}

\begin{abstract}
Background: Pompe disease (PD) is a serious genetic disorder caused by deficiency of acid a-glucosidase (GAA) and subsequent glycogen accumulation inside lysosomes. This study included a cohort of 5 Egyptian infants (1-8 months old) with far lower than average normal GAA activity and clinical signs of PD in 4 of the 5 cases. The fifth case was discovered by newborn screening (NBS). Molecular analysis of the GAA gene was performed to confirm the diagnosis and identify the underlying mutation.

Results: The study identified the causative mutations [c.1193T >C (p.Leu398Pro), c.1134C > G (p.Tyr378*) \& c.1431del (p.lle477Metfs*43)] in 4 cases. However, molecular analysis reversed the expected pathologic state in the fifth infant, where his reduced enzymatic activity was related to the presence of pseudodeficiency allele c.868A > G (p.Asn290Asp) in addition to heterozygous disease-causing mutation c.2238G > C (p.Trp746Cys).

Conclusion: This study presents the first molecular analysis of GAA gene in Egypt and has thrown some light on the importance of PD molecular diagnosis to provide precise diagnosis and enable therapeutic commencement in affected subjects.
\end{abstract}

Keywords: Pompe disease, Acid a-glucosidase, Molecular analysis, Pseudodeficiency allele

\section{Background}

Pompe disease (PD), also called glycogen storage disease type II (GSD II) or acid maltase deficiency (AMD) (MIM\# 232300), is a genetic neuromuscular disorder with an autosomal recessive mode of inheritance. It is caused by mutations in acid alpha-glucosidase gene $(G A A)$ which is localized to chromosome 17q25.2- q25.3 and consists of 20 exons (exon 1 is noncoding). Gene mutations lead to deficiency of GAA enzyme (NP_000143.2) resulting in lysosomal glycogen accumulation in multiple tissues, with profound pathology in skeletal and cardiac muscles [1]. According to the disease age of onset, PD is classified into two main clinical forms: infantile-onset PD (IOPD) which begins within the first months of life, and lateonset PD (LOPD) which appears any time after the first

\footnotetext{
*Correspondence: wessam_sharafeldin@yahoo.com

${ }^{1}$ Medical Molecular Genetics Department, National Research Centre,

Cairo, Egypt

Full list of author information is available at the end of the article
}

year of life. Childhood, juvenile and adult-onset PD are examples of LOPD. IOPD is referred to as classic or nonclassic due to presence or absence of cardiomyopathy, respectively. IOPD is associated with poor prognosis as without treatment most infants die within the first year life. However, LOPD has milder phenotype with more protracted disease course, but eventually leads to respiratory failure [2]. Age of onset, organ involvement and disease severity depend on genotype and residual enzyme activity. Enzyme activity is minimal or absent in IOPD, but may be reduced to varying degrees (3-30\%) in LOPD [3].

PD has been reported in most populations with a worldwide incidence of 1 in 40,000 live births [4]. However, its prevalence may considerably vary among different ethnicities, with a fluctuating range from 1 in 14, 000 in African-American population to 1 in 600,000 in Portuguese population [5]. Moreover, very rare occurrence rate of PD has been documented in some countries such as Sweden and Finland $[6,7]$. 
The spectrum of GAA mutations is very heterogeneous. To date, 648 disease-associated variants have been listed at the Pompe disease GAA variant database (http://www.pompevariantdatabase.nl) [8]. All types of mutations have been described including complex rearrangements; however, missense variations are the most frequent type of mutations ( $50 \%$ ). So far, only one variant in the regulatory region has been described. Although most GAA mutations are restricted to a small number of families, some mutations are frequently reported. Best examples are provided by c.525delT and entire deletion of exon 18 (c. $2481+102 \_2646+31$ del) which are mostly occurring in white populations. Both mutations have been considered as severe variations and are mainly associated with IOPD. Also, the intronic mutation, c.$32-13 \mathrm{~T}>\mathrm{G}$, is the most common mutation in patients with LOPD accounting for about $50 \%$ of cases [9]. Moreover, the variant c. $1935 \mathrm{C}>\mathrm{A}$ is the most frequent in Asia-Pacific [10] and the truncating mutation c. $2560 \mathrm{C}>\mathrm{T}$ was found to be overrepresented in Brazil, North Africa and African-Americans [9].

Different therapeutic approaches have been adopted for treatment of PD, starting from nutrition-based therapy and ending with gene therapy approaches. Nutrition-(low carbohydrate and high protein diet) and exercise-based therapy has been employed as a palliative approach to slow muscle impairment [11]. Gene therapy has been considered especially for treatment of neural manifestations since they are not resolved by peripheral enzyme replacement [12].

In the meanwhile, enzyme replacement therapy (ERT) is the most commonly used therapeutic approach for PD. ERT should be started before presentation of symptoms and occurrence of irreversible injury, to attain optimal treatment. Early intervention of ERT in patients with IOPD markedly achieves improved survival, reduced ventilation need and better movement, resulting in enhanced quality of life [13].

The importance of the effect of early therapeutic intervention in IOPD patients throws the light on the importance of newborn screening (NBS) for early diagnosis and treatment initiation. However, GAA pseudodeficiency alleles can reduce the enzyme activity into the abnormal range interfering with NBS for PD. In this context, gene molecular analysis could be utilized to identify false-positive cases in NBS program for PD [13].

This study was mainly conducted to set up the molecular analysis of GAA in Egypt, where the protocol has been applied on a number of Egyptian patients with reduced GAA enzyme activity.

\section{Methods}

\section{Subjects}

We reported a cohort of 5 subjects with reduced GAA enzyme activity. Biochemical analysis was carried out for all subjects as they exhibited disease manifestations except for one case whose diminished enzyme activity was observed throughout NBS for inborn errors of metabolism (IEMs) carried out in USA.

The study obeyed the beliefs of the Declaration of Helsinki. Written consent was obtained from parents of all enrolled subjects. The study protocol was approved by Medical Research Ethics Committee, Cairo, Egypt (Registration Number: 11098).

\section{Clinical diagnosis}

All subjects were subjected to full history taking, family pedigree construction, clinical examination, and echocardiography.

\section{Biochemical analysis}

EDTA blood sample $(5 \mathrm{ml})$ was collected from each patient. GAA enzyme activity was assayed in mixed leukocytes according to the method of Okumiya et al. (2006), in which 4-methylumbelliferone- $\alpha$-glucopyranoside was used as substrate with an inhibitor acarbose to remove the interference of other glucosidases [14].

\section{Molecular analysis}

Genomic DNA (gDNA) was isolated from peripheral blood leukocytes (PBLs) using the salting out protocol [15]. Primer pairs have been designed to amplify the 19 coding exons of GAA gene and the flanking intronic sequences using the GenBank sequence (accession no. NM_000152.5) (Table 1). PCR was carried out in a total volume of $15 \mu \mathrm{l}$ comprising $100 \mathrm{ng}$ of gDNA, $10 \mathrm{pmol}$ of each primer, $100 \mu \mathrm{M}$ of dNTPs (Thermo Scientific, EU), $1.5 \mu \mathrm{M} \mathrm{MgCl} 2,1 \mathrm{X}$ Taq buffer and 2.5 U Taq polymerase (Thermo Scientific, EU). As a common variation in GAA gene, the entire deletion of exon 18 was firstly tested by amplification of exons 17 and 18 simultaneously using the forward primer of fragment 11 and the reverse primer of fragment 12 . For sanger sequencing, successful PCR products were purified using $4 \mathrm{U}$ Exonuclease I (Thermo Scientific, EU) and 0.4 U Shrimp Alkaline Phosphatase (Thermo Scientific, EU). Then, cycle sequencing PCR was carried out using BigDye Terminator v3.1 Cycle Sequencing Kit (Applied Biosystems, USA), followed by removal of dye terminators using CENTRI-SEP purification spin columns (Applied Biosystems). Sequencing was performed using a 3500 ABI Prism DNA sequencer (Applied Biosystems). Data files obtained from the sequencer were displayed by 
Table 1 Sequences of designed primers, product sizes and annealing temperatures

\begin{tabular}{|c|c|c|c|c|}
\hline Fragment & Exon & Primer sequence & Product size (bp) & Annealing $\left({ }^{\circ} \mathrm{C}\right)$ \\
\hline \multirow[t]{2}{*}{1} & 2 & F: 5'CTTTGA GAG CCC CGT GAG TG 3' & 718 & 63 \\
\hline & & R: 5'TTG TGA GGT GCG TGG GTG TC 3' & & \\
\hline \multirow[t]{2}{*}{2} & 3 & F: 5'TCA GAG AGG CTG AAT GTG CTG 3' & 306 & 60 \\
\hline & & R: 5'GCA CAG AGC CCA GAA CTC AC $3^{\prime}$ & & \\
\hline \multirow[t]{2}{*}{3} & $4 \& 5$ & F: 5' GGT GCG CCT CTT CTG ATA TG 3' & 625 & 60 \\
\hline & & R: 5' GGA AAT GAG TCC TAT GGG CTG 3' & & \\
\hline \multirow[t]{2}{*}{4} & $6,7 \& 8$ & F: 5'TGG GGA GAG AGC CTC AAC TC 3' & 653 & 62 \\
\hline & & R: 5'GCA CAC ACC ACG ATC ATC ATG 3' & & \\
\hline \multirow[t]{2}{*}{5} & 9 & F: 5'GCT GTA CAC ACG CAT GAT GTC 3' & 269 & 60 \\
\hline & & R: 5' GCC TCT GCTTTC TAA CCC CC 3' & & \\
\hline \multirow[t]{2}{*}{6} & $10 \& 11$ & F: 5'TCA GGC TGA GGC TCA GTG GG 3' & 465 & 63 \\
\hline & & R: 5'CAG AGA TGA GGG TGC TAA GTC 3' & & \\
\hline \multirow[t]{2}{*}{7} & 12 & F: 5'TAA CGC CAG CCC CAC AGA GG 3' & 380 & 60 \\
\hline & & R: 5'TCC GCTTTT CCT CCT CCC TG 3' & & \\
\hline \multirow[t]{2}{*}{8} & $13 \& 14$ & F: 5'CAT CAG GTG GCC CAG ACA G 3' & 688 & 62 \\
\hline & & R: 5'GAG AGT CTT GGG TGG GTG G 3' & & \\
\hline \multirow[t]{2}{*}{9} & 15 & F: 5' ATG CTG GGT GGC TGA GAA G 3' & 390 & 59 \\
\hline & & R: 5'CCA GGC CCA AAT GTT GTC TC 3' & & \\
\hline \multirow[t]{2}{*}{10} & 16 & F: 5' CCA GCC CCA TCC CAT TCA T 3' & 298 & 59 \\
\hline & & R: 5'GTT CCT CTT TCC GCC ACC T 3' & & \\
\hline \multirow[t]{2}{*}{11} & 17 & F: 5'AGA TGG AGA GCG TGG TTC C 3' & 287 & 55 \\
\hline & & R: 5' CCC CAG CTC TGC AGT GTG 3' & & \\
\hline \multirow[t]{2}{*}{12} & $18 \& 19$ & F: 5'TAG TTA CTG GCA GCC TGG TG 3' & 821 & 57 \\
\hline & & R: 5' ATC CTC TGT TCC TGG CAT CG 3' & & \\
\hline \multirow[t]{2}{*}{13} & 20 & F: 5' CAC AAT GAC GAC CTC TGA GC 3' & 369 & 56 \\
\hline & & R: 5'GGA ACC AGC CTC CCT CTG 3' & & \\
\hline \multirow[t]{2}{*}{$14^{*}$} & $17,18 \& 19$ & F: 5'AGA TGG AGA GCG TGG TTC C 3' & Wild-type: 1386 & 56 \\
\hline & & R: 5' ATC CTC TGT TCC TGG CAT CG 3' & Mutant: 848 & \\
\hline
\end{tabular}

* Amplification of this fragment was carried out to investigate the entire deletion of exon 18 (538 bp)

Finch TV, version 1.4.0. Query sequences were aligned against those present in BLAST/Basic Local alignment search tool (http://blast.ncbi.nlm.nih.gov) [16].

\section{In silico analysis}

Several in silico tools were employed to predict the effect of the firstly reported missense variation including Blosum62 (Amino acid substitution matrices from protein blocks) [17], MutationTaster (http://www.mutat iontaster.org/) [18], PolyPhen2 (http://genetics.bwh. harvard.edu/pph2/) [19], AGVGD (http://agvgd.hci. utah.edu/agvgd_input.php) [20], SNPs\&GO (http:// snps-and-go.biocomp. unibo.it/snps-and-go/) [21], MUTRED software (http://mutpred.mutdb.org/) [22], SIFT \& PROVEAN (http://provean.jcvi.org/index.php) [23] and Hope (https://www3.cmbi.umcn.nl/hope/) [24].

\section{Results}

Here, we reported a cohort of 5 subjects from 4 unrelated families (3 males and 2 females). All of them showed reduced GAA enzyme activity with mean $=0.47 \pm 0.227$ (normal enzyme activity is " $3 \mu \mathrm{mol} / \mathrm{gpt} / \mathrm{h}$ ). Their ages ranged from 1 to 8 months. All patients showed positive consanguinity except one whose diminished enzyme activity was detected throughout NBS for IEMs without observation of any disease manifestations. Other study subjects showed typical picture of IOPD including cardiomegaly, cardiomyopathy, hepatomegaly, feeding difficulties, failure to thrive, developmental delay, macroglossia, muscle weakness, hypotonia and respiratory distress (Table 2).

The entire deletion of exon 18 was detected in none of the enrolled cases. In 4 patients, sanger sequencing succeeded to identify homozygous pathogenic mutations confirming their diagnosis. The detected mutations 
included a frameshift [c.1431del (p.Ile477Metfs*43)], a nonsense [c.1134C > G (p.Tyr378*)] and a missense [c.1193T > C (p.Leu398Pro)] mutations, where the latter was reported in 2 related patients. Moreover, only one heterozygous mutation was detected in one subject who showed reduced enzyme activity during NBS; however, he was recruited without any disease manifestations. Interestingly, gene molecular analysis identified a pseudodeficiency allele (inherited from father), besides the pathogenic heterozygous mutation (inherited from mother) in this subject explaining the low detected enzyme level and denoting for the importance of gene molecular testing to confirm the diagnosis (Table 3). A novel missense mutation (p.Leu398Pro) has been identified in two siblings and already submitted to the ClinVar database (Fig. 1). Performed in silico analysis favored a pathogenic effect for this novel missense mutation. Importantly, the mutated residue is located in the enzyme catalytic domain (347-726 residues). Possible structural alterations induced by this

Table 2 Enzyme activity and main clinical features in the studied subjects

\begin{tabular}{|c|c|c|c|c|c|c|c|c|c|}
\hline Case no & Sex & Age (month) & Consanguinity \# & $\begin{array}{l}\text { 1,4-a glucosidase } \\
\text { activity }(\mu \mathrm{mol} / \\
\text { gpt } / \mathrm{h})^{*}\end{array}$ & Cardiomyopathy & Hypotonia & Hepatomegaly & $\begin{array}{l}\text { Respiratory } \\
\text { distress }\end{array}$ & $\begin{array}{l}\text { Feeding } \\
\text { difficulties }\end{array}$ \\
\hline 1 & M & 1 & + ve & 0.56 & + & + & + & + & + \\
\hline 2 & $\mathrm{~F}$ & 1 & $+v e$ & 0.32 & + & + & + & + & + \\
\hline 3 & M & 4 & + ve & 0.47 & + & + & + & + & + \\
\hline 4 & $\mathrm{~F}$ & 8 & + ve & 0.4 & + & + & + & + & + \\
\hline 5 & M & 4 & $-v e$ & 0.92 & - & - & - & - & - \\
\hline
\end{tabular}

* $\mu \mathrm{mol} / \mathrm{gpt} / \mathrm{h}$ : micromole/gram protein/hour-normal enzyme activity is '3 $3 \mathrm{~mol} / \mathrm{gpt} / \mathrm{h}$; \# all patients showed positive consanguinity. The only exception is the infant whose diminished enzyme activity was detected throughout NBS

Table 3 Mutations identified in the enrolled subjects:

\begin{tabular}{lllllll}
\hline Case no & Base change & Amino acid change & Exon & Variant type & Allele & Clinical significance \\
\hline 1 & c.1193T $>$ C & p.Leu398Pro & 7 & Missense $^{*}$ & Homozygous & Pathogenic \\
2 & c.1193T $>$ C & p.Leu398Pro & 7 & Missense $^{*}$ & Homozygous & Pathogenic \\
3 & c.1431del & p.lle477Metfs 43 & 9 & Frame shift & Homozygous & Pathogenic \\
4 & c.1134C $>$ G & p.Tyr378* & 7 & Nonsense & Homozygous & Pathogenic \\
5 & c.868A $>$ G & p.Asn290Asp & 5 & Missense & Heterozygous & Pseudo deficiency \\
& c.2238G $>$ C & p.Trp746Cys & 16 & Missense & Heterozygous & Pathogenic \\
\hline
\end{tabular}

*Novel mutation

c.1193 T>C (SCV000998899)

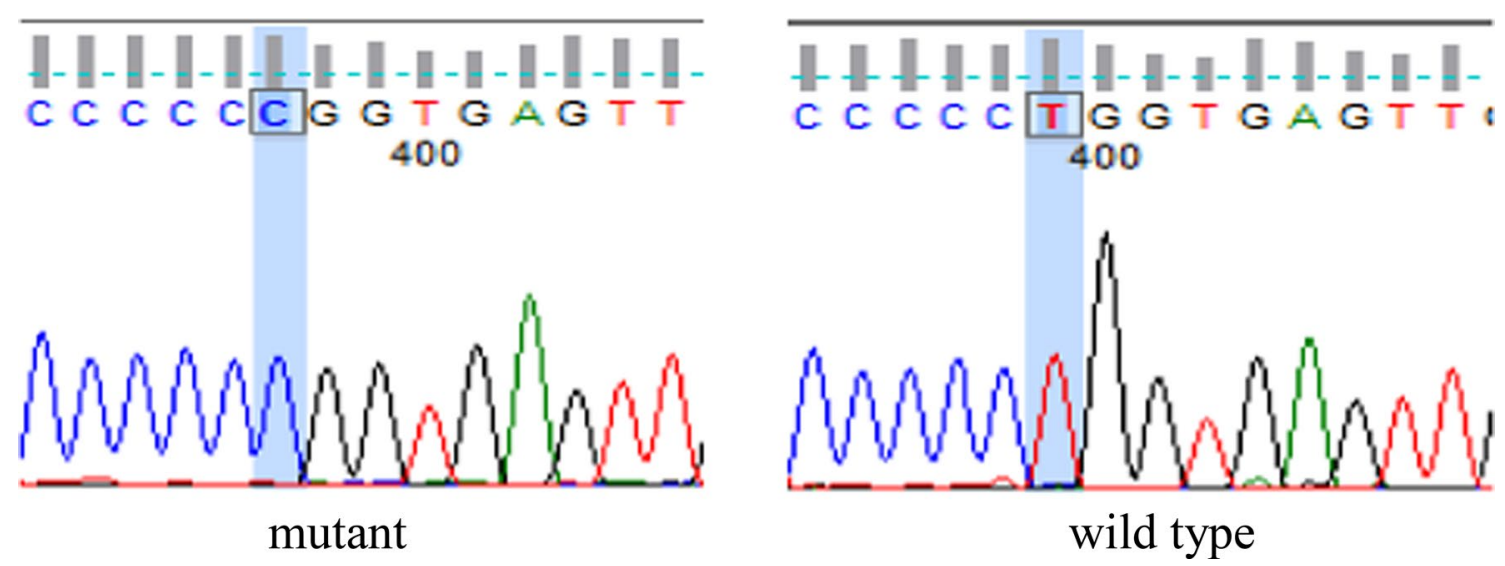

Fig. 1 Sequencing electropherogram of the novel missense mutation. ClinVar accession is written between brackets 
mutation was also assessed using HOPE webserver [24] (Fig. 2). It has been predicted that the mutation will cause an empty space in the core of the protein due to the smaller size of the mutant residue, and this mutation can affect the proper protein interaction and function. It is noteworthy that the used in silico tools also defined the pseudodeficiency allele as pathogenic (Table 4), denoting for the importance of experimental functional analysis to distinguish between pseudodeficiency alleles and real pathogenic mutations.

\section{Discussion}

PD is a multisystemic metabolic disorder caused by deficiency of GAA enzyme leading to progressive accumulation of glycogen inside lysosomes. In turn, this results in lysosomal swelling and rupture all over the human body [25]. It represents one of the most frequent causes of metabolic-based cardiomyopathy in infants [26].

Importantly, PD signs share considerable similarity with several other conditions. Differential diagnoses (DD) of PD mainly include the upper and lower motor neuron diseases. Other DD of IOPD are congenital

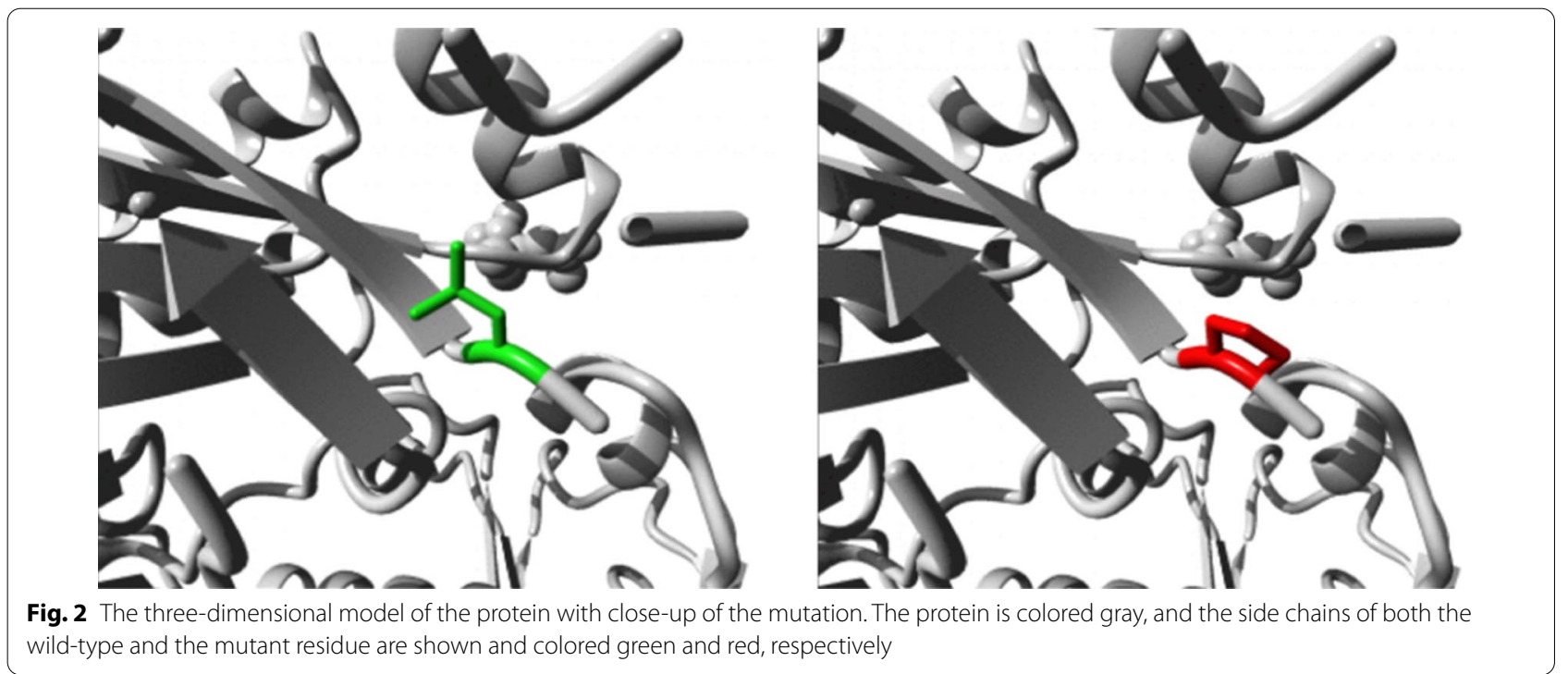

Table 4 In silico analysis for the novel missense variant and the pseudodeficiency allele

\begin{tabular}{lll}
\hline Tool & c.1193 T > C (p.Leu398Pro) & c.868A > G (p.Asn290Asp) \\
\hline MutationTaster (score) $^{\mathrm{a}}$ & Disease causing (98) & Disease causing (23) \\
PROVEAN (score) & Deleterious (-3.108) & Deleterious (-4.525) \\
SIFT (score) & Damaging (0.019) & Damaging (0.009) \\
PolyPhen2 (score) & Probably damaging (0.998) & Probably damaging (1.000) \\
& Sensitivity: 0.27 & Sensitivity: 0.00 Specificity: 1.00 \\
AGVGD $^{\mathrm{d}}$ & Specificity: 0.99 & Class C15 \\
SNPs\&GO (Reliability Index) & \\
MUTRED software (probability) & Class C65 & Disease-related (10) \\
Blosum62 $^{\text {h }}$ & Disease-related (10) & Deleterious (0.595) \\
\hline
\end{tabular}

${ }^{a}$ MutationTaster score ranges from 0.0 (polymorphism) to 215 (disease causing)

b Variants with score $\leq-2.5$ are "damaging" and variants with score " -2.5 are "neutral"

"Variants with score $\leq 0.05$ are "deleterious" and variants with score ' 0.05 are "tolerated"

d PolyPhen2 score ranges from 0 (tolerated) to 1 (deleterious)

e AGVGD classes: C0 (least likely to interfere with function), C15, C25, C35, C45, C55, C65 (most likely to interfere with function)

${ }^{f}$ The SNPs\&GO reliability index extends from 1 (neutral) to 10 (disease-related)

${ }^{g}$ MUTPRED predictions are considered "deleterious" if the score is $>0.5$

${ }^{\mathrm{h}}$ In Blosum62 matrix, a positive score implies that substitution is more likely than any random substitution and vice versa 
muscular dystrophy, spinal muscular atrophy I (acute Werdnig-Hoffman disease), congenital muscular dystrophy, mitochondrial/respiratory chain disorders, glycogen storage diseases type IIIa, idiopathic hypertrophic cardiomyopathy, Danon disease and peroxisomal disorders [27]. It is noteworthy that in the last year, about 25 cases with provisional diagnosis of PD were recruited to our institute for biochemical analysis of GAA. However, low enzyme activity was only detected in the 5 studied patients. This may reflect the phenotypic overlapping of PD with other disease conditions.

This study represents the first report of molecular analysis of GAA in Egypt, in which 5 cases have been reported. Four subjects have been clinically diagnosed with IOPD, showed reduced enzymatic activity and revealed homozygous pathogenic mutations confirming their diagnosis. Mutations in exon 6 were detected in 3 cases from 2 unrelated families. One missense mutation has been firstly reported which could be defined as a disease-causing variation using several in silico approaches. Interestingly, none of the mutations detected in this study has been reported as a frequent pathogenic $G A A$ variation in another population, except for c.2238G $>C$ (p.Trp746Cys) which represented the frequently reported mutation in mainland China accounting for about $27 \%$ of total mutant alleles [28].

One case was included in the study as he exhibited diminished GAA level during enzymatic assay. However, subsequent molecular analysis recommended that he would not develop PD and attributed his low enzymatic activity to pseudodeficiency. This phenomenon occurs due to using synthetic substrates (such as 4-methylumbelliferyl $\alpha$-D-glucoside and 4-methylumbelliferyl$\alpha$-D-glucopyranoside) instead of the natural substrate (glycogen) in laboratory enzyme assay. Enzymes containing pseudodeficiency alleles are known to impair the enzymatic activity on these artificial substrates leading to false-positive results upon enzyme tests. However, they can process natural substrate normally, or at a level that does not result in disease. Interestingly, low enzyme level $(0.36 \mu \mathrm{mol} / \mathrm{gpt} / \mathrm{h})$ was also reported in the subject's father (completely normal) who was found to be homozygous for the pseudodeficiency allele (p.Asn290Asp).

Importantly, p.Asn290Asp variant was firstly reported as a variant with uncertain significance. Later, Kroos and colleagues examined 69 GAA mutations including D290N by site-directed mutagenesis and transient expression in COS-7 cells or HEK293T cells and defined p.Asn290Asp as presumably nonpathogenic [29]. However, in silico tools applied in this study supposed a pathogenic effect for this variant. This in turn denotes for the importance of experimental functional analysis to distinguish between pathogenic mutations and pseudodeficiency alleles, especially in subjects that exhibit none of disease manifestations (Table 3). Generally, a comprehensive NBS program for inherited metabolic disorders is highly recommended worldwide. The key factor is that early diagnosis and management are crucial for the survival and well-being of affected children [30]. Importantly, Egypt and other countries of Middle East have relatively high prevalence for IEMs due to increased rates of parental consanguinity and first cousin marriages [31]. It is noteworthy that national NBS program for congenital hypothyroidism has been already established in Egypt since April 2000 [31] and some pilot studies for expanded metabolic screening have been conducted [32, 33]. As enzyme deficiency can also be reported in healthy individuals due to pseudodeficiency, mutation characterization is a critical step in subjects with decreased enzyme activity to explore whether an enzyme deficiency is due to pathogenic mutation or harmless pseudodeficiency [34].

\section{Conclusion}

This study identified the pathogenic mutations in a cohort of Egyptian patients with PD including a novel missense mutation. On the other hand, it highlights that molecular procedures should be an essential part of the NBS program for PD and other LSDs in newborns who had abnormal results in the biochemical analysis. This provides an accurate diagnosis, relieving parental anxiety in case of false-positive infants and contributing for immediate therapeutic intervention in case of true-positive newborns.

\section{Abbreviations}

AMD: Acid maltase deficiency; GAA: Acid a-glucosidase; GSD II: Glycogen storage disease type II; HGMD: Human Gene Mutation Database; IEMs: Inborn errors of metabolism; IOPD: Infantile-onset PD; JOPD: Juvenile-onset PD; LOPD: Late-onset PD; NBS: Newborn screening; PBLs: Peripheral blood leukocytes; PD: Pompe disease.

\section{Acknowledgements \\ Not applicable.}

\section{Authors' contributions}

EF and EA recruited the samples. EF was also responsible for biochemical analysis. WE and NE performed the molecular analysis and formulated the manuscript under supervision of ME. All authors have read and approved the manuscript.

\section{Funding}

This work was funded by National Research Centre.

\section{Availability of data and materials}

The datasets used and/or analyzed during the current study are available from the corresponding author on reasonable request. 


\section{Declarations}

Ethics approval and consent to participate

Written consent was obtained from parents of all enrolled subjects. The study protocol was approved by Medical Research Ethics Committee of National Research Centre, Cairo, Egypt. Approval Number is 11098.

\section{Consent for publication}

Not applicable.

\section{Competing interests}

The authors declare that there are no competing interests.

\section{Author details}

${ }^{1}$ Medical Molecular Genetics Department, National Research Centre, Cairo, Egypt. ${ }^{2}$ Clinical Genetics Department, National Research Centre, Cairo, Egypt.

${ }^{3}$ Biochemical Genetics Department, National Research Centre, Cairo, Egypt.

Received: 20 April 2021 Accepted: 7 September 2021

Published online: 19 November 2021

\section{References}

1. Nino MY, IntGroen SLM, Bergsma AJ, van der Beek N, Kroos M, Hoogeveen-Westerveld M et al (2019) Extension of the Pompe mutation database by linking disease-associated variants to clinical severity. Hum Mutat 40(11):1954-1967

2. Dasouki M, Jawdat O, Almadhoun O, Pasnoor M, McVey AL, Abuzinadah A et al (2014) Pompe disease: literature review and case series. Neurol Clin 32(3):751-ix

3. van der Ploeg AT, Reuser AJ (2008) Pompe's disease. Lancet (London, England) 372(9646):1342-53

4. Tehrani KHN, Sakhaeyan E, Sakhaeyan E (2017) Evaluation prevalence of Pompe disease in Iranian patients with myopathies of unknown etiology. Electron Phys 9(7):4886-9

5. Almeida V, Conceição I, Fineza I, Coelho T, Silveira F, Santos M et al (2017) Screening for Pompe disease in a Portuguese high risk population. Neuromuscul Disord NMD 27(8):777-81

6. Lindberg C, Anderson B, Engvall M, Hult M, Oldfors A (2016) Search for Pompe disease among patients with undetermined myopathies. Acta Neurol Scand 133(2):131-5

7. Palmio J, Auranen M, Kiuru-Enari S, Löfberg M, Bodamer O, Udd B (2014) Screening for late-onset Pompe disease in Finland. Neuromuscul Disord NMD 24(11):982-5

8. de Faria DOS, in 't Groen SLM, Hoogeveen-Westerveld M, Niño MY, van der Ploeg AT, Bergsma AJ, et al (2021) Update of the Pompe variant database for the prediction of clinical phenotypes: novel disease-associated variants, common sequence variants, and results from newborn screening. Hum Mutat 42(2):119-34

9. Peruzzo P, Pavan E, Dardis A (2019) Molecular genetics of Pompe disease: a comprehensive overview. Ann Transl Med 7(13):278

10. Reuser AJJ, van der Ploeg AT, Chien Y-H, Llerena J Jr, Abbott M-A, Clemens PR et al (2019) GAA variants and phenotypes among 1,079 patients with Pompe disease: data from the Pompe Registry. Hum Mutat 40(11):2146-64

11. Richard E, Douillard-Guilloux G, Caillaud C (2011) New insights into therapeutic options for Pompe disease. IUBMB Life 63(11):979-86

12. Byrne BJ, Fuller DD, Smith BK, Clement N, Coleman K, Cleaver B et al (2019) Pompe disease gene therapy: neural manifestations require consideration of CNS directed therapy. Ann Transl Med 7(13):290

13. Momosaki K, Kido J, Yoshida S, Sugawara K, Miyamoto T, Inoue T et al (2019) Newborn screening for Pompe disease in Japan: report and literature review of mutations in the GAA gene in Japanese and Asian patients. J Hum Genet 64(8):741-55

14. Okumiya T, Keulemans JL, Kroos MA, Van der Beek NM, Boer MA, Takeuchi $\mathrm{H}$ et al (2006) A new diagnostic assay for glycogen storage disease type II in mixed leukocytes. Mol Genet Metab 88(1):22-8
15. Miller SA, Dykes DD, Polesky HF (1988) A simple salting out procedure for extracting DNA from human nucleated cells. Nucleic Acids Res 16(3):1215

16. Camacho C, Coulouris G, Avagyan V, Ma N, Papadopoulos J, Bealer K et al (2009) BLAST+: architecture and applications. BMC Bioinf 10(1):421

17. Henikoff S, Henikoff JG (1992) Amino acid substitution matrices from protein blocks. Proc Natl Acad Sci USA 89(22):10915-9

18. Schwarz JM, Cooper DN, Schuelke M, Seelow D (2014) MutationTaster2: mutation prediction for the deep-sequencing age. Nat Methods 11(4):361-2

19. Adzhubei IA, Schmidt S, Peshkin L, Ramensky VE, Gerasimova A, Bork $P$ et al (2010) A method and server for predicting damaging missense mutations. Nat Methods 7(4):248-9

20. Tavtigian SV, Deffenbaugh AM, Yin L, Judkins T, Scholl T, Samollow PB et al (2006) Comprehensive statistical study of 452 BRCA1 missense substitutions with classification of eight recurrent substitutions as neutral. J Med Genet 43(4):295-305

21. Capriotti E, Calabrese R, Fariselli P, Martelli PL, Altman RB, Casadio R (2013) WS-SNPS\&GO: a web server for predicting the deleterious effect of human protein variants using functional annotation. BMC Genom 14(3):S6

22. Pejaver V, Urresti J, Lugo-Martinez J, Pagel KA, Lin GN, Nam H-J et al (2020) Inferring the molecular and phenotypic impact of amino acid variants with MutPred2. Nat Commun 11(1):5918

23. Choi Y, Chan AP (2015) PROVEAN web server: a tool to predict the functional effect of amino acid substitutions and indels. Bioinformatics 31(16):2745-7

24. Venselaar H, te Beek TAH, Kuipers RKP, Hekkelman ML, Vriend G (2010) Protein structure analysis of mutations causing inheritable diseases. An e-Science approach with life scientist friendly interfaces. BMC Bioinf 11(1):548

25. Thurberg BL, Lynch Maloney C, Vaccaro C, Afonso K, Tsai AC-H, Bossen E, et al (2006) Characterization of pre- and post-treatment pathology after enzyme replacement therapy for pompe disease. Lab Investig 86(12):1208-20

26. Fateen EM, Hamza HS, Matty DMA, Gouda AS, El-Saiedi SA, Saleh SM, et al (2017) Biochemical study of glycogen storage disease type II (Pompe Disease) in Egyptian infants: Wolters Kluwer Health | Lippincott Williams \& Wilkins

27. Kishnani PS, Steiner RD, Bali D, Berger K, Byrne BJ, Case LE et al (2006) Pompe disease diagnosis and management guideline. Genet Med $8(5): 267-88$

28. Liu X, Wang Z, Jin W, Lv H, Zhang W, Que C et al (2014) Clinical and GAA gene mutation analysis in mainland Chinese patients with late-onset Pompe disease: identifying c.2238G > C as the most common mutation. BMC Med Genet 15:14

29. Kroos M, Hoogeveen-Westerveld M, Michelakakis $H$, Pomponio R, Van der Ploeg A, Halley D et al (2012) Update of the pompe disease mutation database with 60 novel GAA sequence variants and additional studies on the functional effect of 34 previously reported variants. Hum Mutat 33(8):1161-5

30. Pourfarzam M, Zadhoush F (2013) Newborn screening for inherited metabolic disorders; news and views. J Res Med Sci 18(9):801-8

31. Saadallah AA, Rashed MS (2007) Newborn screening: experiences in the Middle East and North Africa. J Inherit Metab Dis 30(4):482

32. Hassan F, El F, Mandour I, Hady S, Selim L, Abdel S et al (2009) Preliminary results of Egypt experience for use of tandem mass spectrometry for expanded metabolic screening. J Appl Sci Res 5:1425-35

33. Hassan FA, El-Mougy F, Sharaf SA, Mandour I, Morgan MF, Selim LA et al (2016) Inborn errors of metabolism detectable by tandem mass spectrometry in Egypt: the first newborn screening pilot study. J Med Screen 23(3):124-9

34. Bronner P, Rodier G, Cohen E, Derouiche F, Boulay C, Courtois S (2000) Lysosome enzyme pseudodeficiency. Revue neurologique 156:1005-12

\section{Publisher's Note}

Springer Nature remains neutral with regard to jurisdictional claims in published maps and institutional affiliations. 